\title{
Supporting Older Adults Using Privacy-Aware IoT Analytics
}

\author{
Mateusz, Mikusz, Nigel Davies, Peter Shaw, Christopher Bull, Bran Knowles, Niall Hayes, Lucas Introna \\ Lancaster University, $U K$ \\ $\{$ m.mikusz, n.a.davies, p.shaw, c.bull,b.knowles.1,n.hayes, l.introna\}@lancaster.ac.uk
}

\begin{abstract}
Mobile computing and the IoT offer the potential to provide new applications to support our ageing population. However, there has been little research into the implications for system design of supporting this specific user demographic. In this paper we consider these implications, focusing in particular on an architectural design that offers the potential for complex cross-device analytics while meeting the privacy and usability concerns of older adults.
\end{abstract}

Keywords: internet-of-things, analytics, mobile applications, older adults

\section{Introduction}

Improvements in food, health and hygiene are delivering a world with an increasing number of older adults. In parallel, society is becoming increasingly dependant on technology with many public and private services such as shopping, banking and civic interactions migrating on-line. While many services are aimed (implicitly or explicitly) at younger demographics, their importance to older adults cannot be overstated.

Mobile devices would appear to offer an ideal route for older adults to access digital services. In particular, the portability, low-cost and controlled nature of most mobile platforms lend themselves to widespread adoption by the general public. As a result, there has been significant research into designing systems (fixed and mobile) that address the physical, cognitive and sensory needs of older adults [16, 2, 3]. However, to date the vast majority of this research has focused on HCI issues [16] e.g. creating new UI widgets for those with limited handeye coordination. Such initiatives have had limited success, with many of the research challenges that existed 20 years ago continuing to be re-explored [7].

In this paper we choose not to explore UI issues for older adults but instead seek to address the broader system implications of designing for this user group - covering systems that include both mobile and IoT elements. Our analysis is baaed on a series of engagements with older adults in which we co-created new public-service applications designed to reduce social isolation. Many of the design issues that arose during these engagements were associated with system concerns such as security, disconnection and data availability, in addition to usabil- ity considerations. In addition to describing an overall architectural approach we focus in particular on supporting mobile and IoT analytics are able to capture how such systems are being used by their target community. For example, the IoT can be used to identify opportunities to improving the efficiency of council services for older adults, automatically detecting needs based on their behavioural patterns. In order to understand how users (in particular, older adults) interact across digitised environments, novel forms of IoT analytics systems will be required that extend well beyond traditional Web analytics and seek to capture data on a wide range of user interaction points including interactions with IoT devices in the real world, phone calls and face-to-face meetings, social media activity and Web activity, as well as additional events in the real world (e.g. collection of a prescription from a pharmacy and environmental sensing data) where such data will be of particular use to support older adults. A specific challenge is how to instantiate such comprehensive analytics tracking and monitoring capabilities whilst respecting user privacy - an issue of particular importance to older adults.

Our key contributions are:

1. the identification of a set of design considerations for mobile and IoT computing systems targeted at older adults,

2. a prototype architecture that embodies these considerations with a particular focus on supporting domainspecific analytics.

3. a set of novel analytics reports co-created with council workers and third-parties and specifically designed to enable the tracking of specific usage patters across applications.

\section{The Mobile Age Ecosystem}

The work described was conducted in the context of Mobile Age, a project that aims at designing and developing technology to support older adults in their day-to-day living and address issues of, for example, social isolation and loneliness. In this section, we describe the methodological approach of Mobile Age, the resulting Mobile Age ecosystem and a set of scenarios to illustrate the use of technology designed for older adults. 


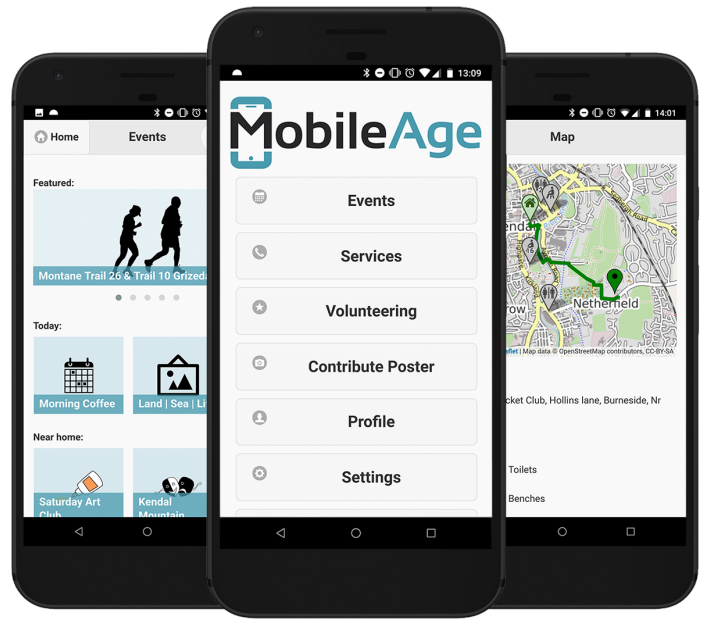

Fig. 1: User interface of Mobile Age App.

\subsection{Methodology}

In the context of Mobile Age, we conducted a series of interviews and co-creation workshops in order to capture a set of design considerations for the design and development of applications specifically targeted to support older adults and use IoT analytics to further improve the user experience and quality of the applications developed.

In particular, we first conducted a total of 25 preliminary interviews and workshops with older adults (representing the target demographic of end-users) and the wider 'senior services' community (representing stakeholder groups that will access novel analytics insights) providing us with a better understanding of older adults' lived experiences. Following these interviews we conducted an additional four workshops that explored these themes in more detail - including access to public services and tackling social isolation. Through these workshops, we were able to identify design considerations and requirements for potential apps that support older adults (e.g. the importance of face-to-face activities, public transport routes and high interest in serendipitous events). Based on the interviews and discussions in workshops, we created a set of scenarios to visualise the potential both of applications designed to support older adults and IoT analytics that can be used to improve the quality of services.

Following our preliminary interviews and workshops, we conducted an additional set of 12 workshops in which we created prototypes of mobile applications for older adults that focus specifically around local events and transport. Additionally, we extracted key features for our applications by further exploring live experiences of older adults in the context of the themes identified above. For example, our workshop participants highlighted the need to support personalised transport options that reflected individual concerns (e.g. ensuring that any journey has a specified upper bound of walking time).

\subsection{Resulting System}

Based on the co-creation workshops, we designed and developed Mobile Age App - an application for mobile phones and tablets that has been specifically designed to encourage independent living by tackling the problem of social isolation. In particular, groups of older adults identified challenges in finding information regarding local events and determining their accessibility (e.g. suitable transport options). In order to address these challenges, Mobile Age App is comprised of two main features: 'Events' and 'Transport' (fig. 6). The 'Events' component allows older adults to search for events taking place in their area. The events are typically populated by council workers and volunteers who collect relevant and appropriate events for the target demographic. In order to support older adults in finding appropriate transport options to these events, we additionally integrated the ability to search for transport options such as buses and trains. Furthermore, additional components have been included such as 'Services' (access to council services and information such as guides to avoid scams), 'Volunteering' (local volunteering opportunities), 'Profile' (configuring preferences such as preferred transport modalities), and 'Settings' (configuring the behaviour of the app such as the level of analytics tracking).

\subsection{Analytics Scenarios}

We developed a set of scenarios to illustrate how Mobile Age App in combination with IoT-enabled analytics can be used to support older adults - both by council workers or third-parties using analytics insights to improve the service, and by automatically feeding analytics data back to the user in order to provide smarter services such as recommendations based on their usage patterns.

\subsubsection{Scenario: Public Transport}

The first scenario describes the use case in which users of the app are seeking local events in the area.

Barbara is using a MobileAge app to find social events in her local area. She discovers an afternoon tea session, held in the town hall, that she is interested in attending. Whilst viewing the details of the event in the app, she selects an option to pre-book a bus ticket - this opens a separate MobileAge app for booking public transport. Barbara searches for buses to and from the event's location; she sees that there is a direct bus during the day, but for the journey back at night there is only one route available that takes longer and requires changing buses in an adjacent town. Barbara decides not to attend the event and finds a different one she is less interested in. She purchases a bus ticket, closes the app, then puts her tablet away. She shortly receives an email confirming the purchase.

The available datasets to support analytics include information about local social events that were browsed in an app and insights on the intent to purchase transport to the event (selecting 
the option to pre-book a bus ticket). In a separate public transport app, there is data about journeys (pickup location, destination, and times), whether a bus route is available, and whether a purchase was made. There is also external data available, provided by the local bus company, on ticket sale stats, and bus route delays.

In addition to end-users, the scenario described provides opportunities for council workers and third-parties to gain valuable insights.

Council person Joe is interested why certain council run events have better participation than others. Some of the organisers know anecdotally that some dates, times, and places are better for attendance than others - Joe would like to explore and better understand why this may be. Joe looks at analytics reports and sees patterns of people using the social events app, navigating to the separate transportation app, but then not following through with a ticket purchase.

The opportunities for analytics include insights as to whether end-users accessed the 'local events' app, and how the travel application was used to organise transportation to and from events that have been viewed previously. Using unique user profiles and identifiers will enable analytical insights that span across multiple applications within the Mobile Age ecosystem. By including data from external entities (such as ticket purchase statistics from private transport companies) the accuracy of analytics can be increased.

\subsubsection{Council Telephone Query}

Margaret is moving to a new house and wishes to know when her bins will be collected at her new property. Without hesitation, she picks up her phone, searches for the local council's telephone number and makes the call. She is met with courteous service, and at the end of the call she is informed that the information she required could be found on the council?s website - she shrugs this off with "but I don't know where to look for it".

The available datasets in this scenario include Margaret's phone call records (in order to identify whether a call has been placed to the council) and activities that potentially led up to the call. Additional analytics data can also be collected from the council's website.

Jane works at the council and is considering customer experiences of people who call the council. She is particularly interested in exploring if information for common queries can be easily found on the council's website, or why people fail to find these answers before making a call. She can view aggregated reports that show that many people act as Margaret did - calling the council first without ever exploring the web site.

The opportunities for novel analytics reports include insights into the proportion of people who call the council after first trying to find an answer to their query on the council website or the Mobile Age app. Analytics data captured on the user's mobile device can be correlated with data captured through Web analytics in order to understand users' navigation patters prior to calling the council, e.g. to provide insights on which activity or page lead the user to the call. Councils can utilise this information in order to improve Web sites and navigation, or determine if someone revisited the Web site in future and found the information needed.

\section{System Design Considerations}

Through our formative, co-design and current build phases we have begun to establish an understanding of the unique challenges, needs and wants of older adults, and the potential role of mobile-supported public services to address the growing problem of social isolation. While many of the issues raised relate to the specific applications and interaction patterns we are co-creating, what is striking is the emergence of a number of general design considerations that relate to more fundamental systems-level issues.

\subsection{Usage Models}

The number of smartphone users amongst adults aged 65-74 in the UK has risen $11 \%$ in the last year (from $28 \%$ in 2015 to $39 \%$ in 2016) while tablet use in adults aged $75+$ rose from $15 \%$ to $27 \%$ [15]. However, over-65s spent half the amount of time online compared to $16-24$ year olds. While this may be down to the greater number of online services younger adults engage with such as social media, evidence from our formative studies have identified distinctive models of mobile device use.

\subsubsection{On-demand Interactions}

Our older adults described many situations where mobile devices were used on a 'on-demand' basis. That is, mobile devices were accessed (or even powered on) only when they needed to use a mobile service, such as calling a relative or checking email once they were made aware that an email had been sent to them. This is in contrast to other user groups where common mobile usage models involve 'always-on' interactions, and where devices are often considered an extension of self [19].

In designing for older adults it is therefore critical that services account for 'on-demand' usage models - impacting on a broad range of system aspects from how to deliver system updates to techniques for attracting user attention.

\subsubsection{Supporting Disconnected Operation}

The issue of internet connectivity was highlighted as a major barrier for our older adults to access online services. Although this was not completely unexpected given that $3 \mathrm{G}$ mobile networks covered only $29 \%$ of rural areas [14], what was unexpected were examples of 'in-home' disconnectedness where participants did have WiFi but due to the combination of poor signal propagation and limited personal mobility (e.g. the user had difficulty walking around the house) loss of connectivity 
was common when attempting to use mobile devices. Consequently, while the mobile research community may perceive connectivity as a diminishing challenge, our unique experiences have highlighted that for older adults issues of disconnection remain commonplace, and designers must consider the usage model constraints of older adults when developing services, and avoid connectivity assumptions. Our conceptual design work on 'personal data containers' [13] described in section 4 aims to specifically consider issues of disconnected operation through a localised, personal mobile data store that can facilitate access to data across multiple mobile applications to ensure particular levels of service operation.

\subsection{Trust and Security}

Our experiences with older adults has underlined the important role social-support networks play in enriching their daily lives (e.g. personal health, mobility). A key characteristic of these networks, and the day-to-day relationships older adults share with family members and caregivers is an established level of trust to provide reliable assistance when needed. Nevertheless, from our understanding of current approaches to public mobile service provision, very few have begun to consider how this trusted resource can be exploited to address common problems of mobile service interaction, and enhance accessibility to trusted relationships to support more effective engagement within the digital environment.

\subsubsection{Supporting Flexible Models of Trust}

A clear consideration therefore must be given to the way in which trust relationships in the physical world can be identified, formulated and represented in the digital environment. For example, a common scenario discussed by our older adults during co-creation activities was the inability to access trusted support when using mobile services. Furthermore, participants discussed occasions where they required assistance with their computer but would have to wait until a family member would visit in order to access help.

\subsubsection{Explicit Data Ownership}

Security and the protection of personal information was identified as a major concern for our participants, particularly when sharing information (e.g. bank details) with services via mobile devices. For example, it was common for our participants to hesitate or refuse to provide basic information when interacting with mobile web services, such as submitting their postcode for local searches on popular car buying websites. In particular, participants highlighted specific concerns about how data collected about them was managed (e.g. where it was stored) and issues of ownership once submitted.

While we recognise the technical challenges of mobile application security and data privacy are well understood, for older adults the perceived mis-trust in digital services is predominantly based on a limited understanding and awareness of how their data is managed and used. It is therefore critical that designers begin to consider mechanisms for enabling older adults to feel more in control of their personal data. This consideration was underlined during discussions with participants and their desire to retain personal data locally on the device rather than in the cloud.

\subsection{Adoption}

Technology adoption is influenced by a number of factors (e.g. usefulness, cost) across the general population. Although, for older adults additional factors such as physical capability and familiarity with new technology are often overlooked by designers [9]. From our formative discussions a number of specific design considerations were identified that aim to address issues of user adoption and acceptance.

\subsubsection{Supporting Face-to-Face Interactions}

The importance of social, face-to-face interaction was emphasised by a number of participants. Our discussions highlighted numerous examples where face-to-face interaction was perceived to be more valuable than alternative forms of communication (e.g. voice calls, text), and was considered highly beneficial in addressing feelings of social isolation. Indeed, many participants perceived a growing disconnection with their local communities primarily due to the closure of traditional amenities (e.g. post office, banks) that for the majority of their lives had represented hubs for social engagement. Clearly, as local amenities and public support services move online designers must consider alternative approaches to service provision that are sensitive to the needs of older adults. Our older adults underlined their concern in adopting technology that further contributed to the reduction of face-to-face communication. However, they acknowledged that digital communication capabilities would be good for people who are house-bound, but highlighted fear over the introduction of technology in every aspect of their lives.

\subsubsection{Reducing the Burden of Use}

Participants unwillingness to adopt new technology was motivated by a general perception that technology use is difficult, demands concentrated effort and complicates the execution of everyday tasks. As part of our co-design process we chose to investigate the "why should I bother" mentality identified with many participants by considering the potential of mobile service personalisation. For example, participants that had issues with eye-sight were taken through a speculative design of a mobile service that personalised content by using audio to playback information. Our older adults felt personalisation had potential in addressing the perceptions of complexity and effort. 


\subsubsection{Invisible Assistance}

We discovered a broad range of attitudes towards the adoption of technology that specifically targeted perceived constraints (e.g. cognitive limitations such as forgetfulness) of older adults. Indeed, the default assumption amongst most technology advocates and designers would be that explicit technology support to offset user constraints and maximise usability of digital public services would be welcomed. However, while many of our older adults expressed excitement at the possibility of mobile services that seamlessly adapt to their needs, others were unsettled by the thought of services actively assisting them differently due to their demographic profile. Designers may wish to consider more sensitive approaches that aim to provide proactive assistance while not explicitly outlining applications and features for older adults.

\subsection{Analytics Reports}

In addition to end users of Mobile Age App, we have also captured a set of requirements from council workers and thirdparties specifically to help us design the IoT analytics platform. The requirements are primarily focussed around three overarching themes: Privacy, Analytics Reports and Insights and Tracking and Monitoring of older adults.

\subsubsection{End-user Activity}

Stakeholders were particularly interested in analytics reports that visualise activity trails of users beyond the interactions on the mobile phone application itself. For example, users can use Mobile Age App to view and register to local social events - and additionally find appropriate public transportation in order to attend the event. Gaining insights into the behaviour of users, e.g. understanding why certain events received less registrations in relation to others, or why users registered to an event decided to not attend. Analytics reports could provide insights into the interactions of an user after registration, or shortly prior to the event (e.g. requesting transport options prior to an event which the user has not attended could suggest issues with public transportation options).

In addition to tracking users beyond the application, stakeholders mentioned traditional application areas of analytics in the context of Mobile Age App. In particular, the stakeholders hope to use analytics in order to "improve how events are published" (P1) or "use to improve the app [Mobile Age App] itself" (P1) - i.e. using analytics to improve the user experience and quality of both the application itself and the content published through Mobile Age App.

\subsubsection{Tracking and Monitoring}

Participants explicitly mentioned two distinct purposes for analytics: tracking of user interactions and monitoring of older adults.

In order to support a comprehensive tracking of user interactions for the scenarios described above (in particular Council
Telephone Query), an analytics system is required to be able to track users across different domains, locations and applications. For example, in this case analytics events from Mobile Age App need to be combined with telephone records from the user's device and the council. As a response to this scenario, council workers described the application as "very useful" (P1).

As an extension to tracking of user interaction, participants raised the monitoring of older adults as an alternative application scenario. Users with difficulties could have the option to opt in to comprehensive monitoring "with their participation and agreement" (P2). Analytics would be used to orchestrate data from distinct sources such as wearable and "peripheral devices" (P2).

The participants mentioned specific behaviours that need to be considered in the design of the system. Users may not always have access to the internet or carry their devices - putting on limitations on the ability to track users' locations to, for example, determine their attendance at specific events.

\subsubsection{Supporting Privacy}

A future IoT analytics system that is designed to support the scenarios described will potentially consist of comprehensive tracking and monitoring capabilities of end users. A number of concerns have been raised by participants regarding the level of tracking and lead to a set of design considerations in order to address privacy-related concerns - particularly in light of recent GDPR. The participants particularly evaluated the case in which Mobile Age App and the corresponding analytics module captures and combines user-related data across devices and application domains (e.g. by correlating telephone queries with Mobile Age App interactions and Web site visits).

The participants believe that transparency regarding the communication of the level of tracking is key in this application domain - and users need to be informed about analytics taking place. Additionally, user's consent to location and interaction tracking needs to be obtained in any case, e.g. to be able to track and correlate hospital appointments or registrations to social events.

\section{System Architecture}

Our proposed architecture is comprised of three core components designed specifically to encourage use by older adults (fig. 2): an analytics framework that enables widespread data collection yet addresses privacy concerns raised by older adults, a data storage and exchange facility that aims to lower the barrier of use while providing a security model that reflects older adults' ways of working, and a unified application framework that provides "a walled garden" that helps reassure older adults of the trustworthiness of applications. 


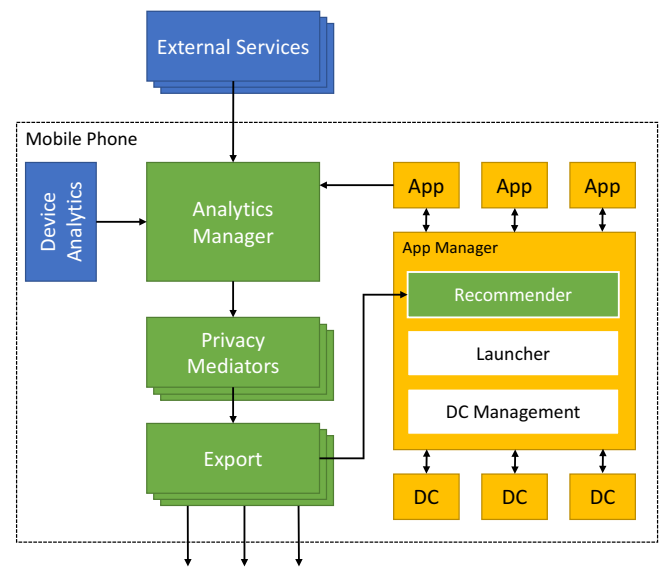

Fig. 2: High-level architecture consisting of an analytics framework (green), external data sources (blue), and application and data store management (yellow).

\subsection{Mobile Client}

The Mobile Age App is the main gateway for end-users in order to access services and obtain relevant information on events and other social activities. The Mobile Age App is comprised of three core components: Launcher, Data Container Management, and Recommender. The Launcher provides access to individual services and applications that have been tailed towards the needs and requirements of older adults and is the component most visible to the end user. In order to address requirements regarding trust and security (sec. ??), we included support for data containers. Each application part of Mobile Age App has access to a distinct Data Container in order to store user-related information, and data cannot be shared beyond individual Data Containers without user consent. The Recommender component connects the launcher with the analytics system in order to learn about the behaviour of the end user, and provide recommendations on potentially relevant and interesting applications and services based on the users behaviour.

\subsection{Analytics Backend}

In order to address requirements and concerns raised during the co-creation workshops regarding privacy, trust and security, we developed an analytics architecture that collects and processes data related to a user on their personal mobile device. This analytics architecture supports data captured by the device's operating system, applications running under the App Manager umbrella and analytics data about the user that was originally captured outside of the mobile device, enabling the correlation of user-related datasets without the need for uploading any personal and sensitive datasets into other services. In addition, the analytics system utilises the concept of Privacy Mediators [1] allowing users to control the level of sharing of their personal data and providing a high level of confidence and control about the ownership and use of personal data. The analytics framework consists of four main components: a set of Data Sources, the Analytics Manager, Privacy Mediators, the Export module and the Recommender.

Data Sources. The analytics framework accesses four distinct classes of data source: external services (e.g. statistics on contacting council services), device-related logs (e.g. usage statistics), events from applications that are running within our platform (e.g. interaction patterns), and user profiles stored as part of the applications (e.g. user preferences). To be able to import data from these external sources, our framework features a set of import modules each of which are specific to the data source and which implement the relevant application programming interface to connect to the external service (e.g. accessing and parsing phone records).

Analytics Manager. The analytics manager orchestrates import modules, the combination of user-owned datasets (both from internal and external sources) to gain new analytical insights, and the privacy mediator pipeline. It further provides a set of application programming interfaces for applications running within our platform to report relevant events and request information through the Recommender.

Privacy Mediators. Due to the high sensitivity of the collected datasets, the privacy mediator component is designed to address issues in trust and data privacy. Prior to the export and use of any dataset, it is routed through a pipeline of userspecified privacy mediators that restrict the access to datasets both in terms of access control and level of information sharing. Example mediators could provide features to remove useridentifiable information prior to export, and define levels of redaction.

Export. To allow third party services or other users to gain a better understanding on interaction events of a person, the export component enables the specification of 'injection modules' that post analytical insights in compliance to user configuration and privacy mediators to both external services as well as applications that are part of our application framework.

Recommender. The Recommender component provides a way to feed analytics insights back into applications by enabling developers to specify modules that request relevant datasets from the Exporter (i.e. upon passing through the privacy mediator pipeline), and make these available to applications in the form of recommendations, e.g. recommending users access new services based on prior interactions.

\subsection{Implementation}

The Mobile Age App has been implemented on top of Cordova and deployed in the Google Play Store and Apple App Store. As part of the Mobile Age App, we deployed a number of services integrated in the application: Events (local events in the area), Services (access to council services such as bin collection times), Volunteering (local volunteering opportunities available in the area), Contribute Poster (users can contribute images of posters that may be of interest to other users), Profile (configuring preferences such as preferred transport modali- 
ties), and Settings (configuring the behaviour of the app such as the level of analytics tracking).

Whilst conceptually, the IoT Analytics System is part of the Mobile Age App, we have chosen to implement analyticsrelated features as part of a cloud service with a basic set of RESTful application programming interfaces - this enables us to reduce the load on the client devices but care will need to be taken to ensure that the provision of this functionality in the cloud (albeit within the user's domain rather than the service provider) is compatible with older-adults' privacy concerns. The system has been implemented entirely in Python on top of the Django Web framework. In addition, we developed a set of client libraries (built based on Pheme's client libraries [12]) for Python, PHP and JavaScript in order to allow third-parties to push relevant data into IoT Analytics System. We note that the Recommender component has not yet been implemented but will form a significant portion of our future work.

\section{Example Reports}

We developed a set of analytics reports that help both developers and council services to understand how older adults can navigate and interact with the application and external services. In this section, we provide a set of example reports to illustrate the applicability of IoT Analytics System.

\subsection{Usage and Activity}

\section{Users}

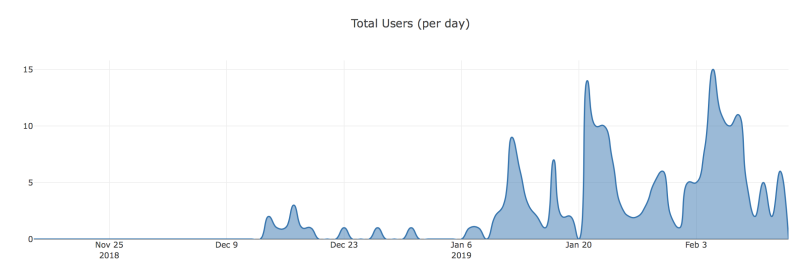

Fig. 3: Active users of Mobile Age App.

The first set of reports are similar to existing mobile and web analytics and focus on visualising interactions of end users with Mobile Age App. Fig. 3 is an example report that provides insights into the number of active users per day allowing stakeholders to understand the uptake of the application among older adults in their area. Note that in contrast to many existing web analytics systems this report also includes data captured when the device was being used off-line.

Fig. 4 provides insights into the types of user interaction events captured by IoT Analytics System. For example, it shows users adding events to their calendar, searching for transport modes and signing up to local events. Such usage and activity reports are particularly relevant to both developers and council workers giving insights on the uptake of the application, capturing popular services within the application giving insights into potential issues with the app.

\section{Event category break-down (by day)}
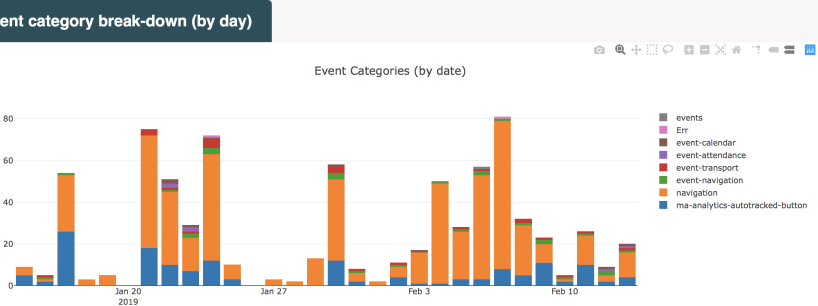

Fig. 4: Individual interaction events.

\subsection{Event Attendance}

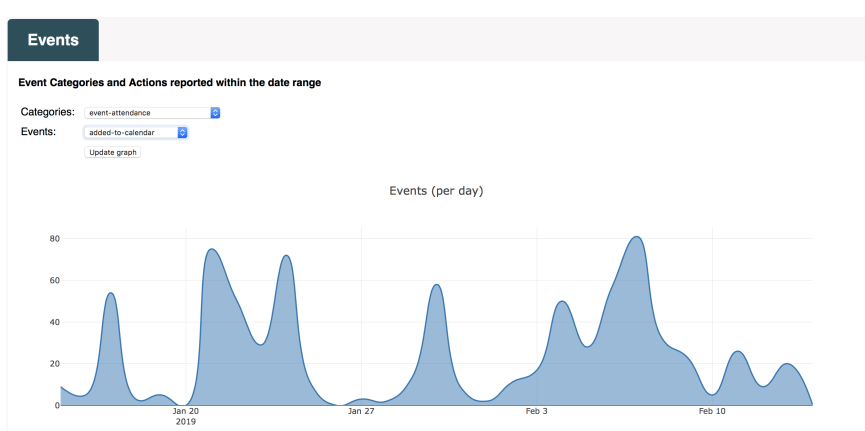

Fig. 5: 'Add to calendar' interactions for local events.

As part of the initial scenario (sec.2.3.1) and stakeholder discussions (sec. 3.4.1) support for tracking event attendance and user activity before and after signing up to events has been expressed as a valuable insight. In order to address this requirement, we developed reports that provide detailed insights into user activity in Mobile Age App relating to event sign ups. For example, fig. 5 captures users adding events to their mobile phone calendar - which can be used as an indication for the (expected) attendance at events.

\subsection{Public Transport}

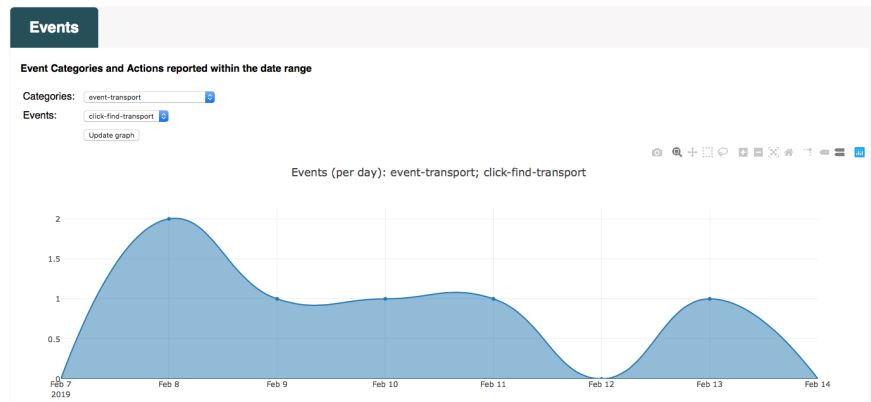

Fig. 6: Public Transport.

Understanding the preferred transport choices of older adults is important for councils in order to understand, for example, if the available transport has an impact on event registrations and attendance. The Mobile Age App is capable of tracking users 
searching for transport options in the app. Linking such activities with the actually available transport options, and real-time departure data of local transport can provide further insights into the possible reasons why, for example, older adults have chosen not to attend an event after initially signing up for it.

\section{Related Work}

Co-creation and participatory design approaches have been successfully used for designing mobile systems for older adults, e.g. [8] and [11]. Design guidelines and checklists have been produced for the design of mobile phones for older adults [16] but have not considered broader systems issues. The diversity amongst older adults presents challenges in designing with them, as well as for them and [3] discusses elements of diversity (such as vision or memory), reflecting on including them in design processes, and the impact on design decisions. More generally, there has also been work on designing mobile applications for specialist groups, e.g. children [4] and autism sufferers [6].

The concept of developing mobile phone applications within a cross-platform compatible framework is often referred to as a "Unified Mobile Platform" and is offered by commercial providers to simplify the development of mobile applications. Ex Libris campusM is a commonly used platform among universities that features a set of sub-applications such as timetables [10]. Through a set of unified JavaScript APIs developers can extend the functionality of campusM and implement custom applications using Web technology. The platform handles the implementation of the APIs on the operating system level-allowing developers to build out cross-platform compatible applications.

We note that campusM (and similar systems such as Firebase [5]) are designed to be used by single stakeholders and organisations that implement and deploy (sub-) applications. In contrast, our platform is designed to allow distinct developers and services to build mobile applications under a common umbrella whilst keeping data secure and private and providing ways to share datasets across applications locally on the device.

Significant work has been done in combining analyticsrelevant data on mobile phones and the fusion of mobile phone sensor data predominately for behaviour and activity recognition [18]. Researchers have additionally used datasets from external services to enable the development of context-aware applications. For example, Phan et al. [17] developed a mobile phone framework that integrates data from internal phone sensors and the users social media accounts. Similar to campusM, developers access sensor data through high-level Web technologies including JavaScript and are not required to implement APIs on the operating system level.

\section{Concluding Remarks}

Mobile devices and the IoT have the potential to significantly improve the quality of life for older adults. However, much of the research in this area has focused on simple changes to application user interfaces in order to improve accessibility. In contrast we seek to explore how catering for this specific demographic impacts on the underlying system architecture for such applications. Drawing on an extensive co-creation exercise we have reported on a set of design considerations for systems to support older adults adults - focusing in particular on how to support the type of complex analytics reports that will be required to improve overall system performance.

\section{Acknowledgments}

The Mobile Age project has received funding from the European Union's Horizon 2020 research and innovation programme under grant agreement No 693319 (Mobile Age).

\section{References}

[1] Nigel Davies et al. "Privacy Mediators: Helping IoT Cross the Chasm". In: Proceedings of the 17th International Workshop on Mobile Computing Systems and Applications. HotMobile '16. St. Augustine, Florida, USA: ACM, 2016, pp. 39-44. ISBN: 978-1-4503-4145-5. DOI: $10.1145 / 2873587.2873600$, URL: http : / / doi.acm.org/10.1145/2873587.2873600

[2] Miranda A. Farage et al. "Design Principles to Accommodate Older Adults". In: Global Journal of Health Science 4.2 (2012). DOI: 10.5539 / g jhs . v4n2p2

[3] Peter Gregor, Alan F. Newell, and Mary Zajicek. "Designing for Dynamic Diversity: Interfaces for Older People". In: Proceedings of the Fifth International ACM Conference on Assistive Technologies. Assets '02. Edinburgh, Scotland: ACM, 2002, pp. 151-156. ISBN: 158113-464-9. DOI: 10 .1145/638249.638277. URL: http : / / doi . acm . org / 10.1145/ 638249.638277 .

[4] Fabian Hemmert et al. "Co-designing with Children: A Comparison of Embodied and Disembodied Sketching Techniques in the Design of Child Age Communication Devices". In: Proceedings of the 9th International Conference on Interaction Design and Children. IDC '10. Barcelona, Spain: ACM, 2010, pp. 202-205. ISBN: 978-1-60558-951-0. DOI: 10 . 1145/ 1810543 . 1810571. URL: http : / / doi . acm . org/10 . $1145 / 1810543.1810571$.

[5] Google Inc. Firebase. 2017. URL: http : / / firebase.google.com (visited on 10/19/2017). 
[6] Muhamad Fairus Kamaruzaman et al. "Developing User Interface Design Application for Children with Autism". In: Procedia - Social and Behavioral Sciences 217.Supplement C (2016), pp. 887 -894. ISSN: 18770428. Dor: https : / / doi org / 10 .1016/j. sbspro.2016.02 .022, URL: http: / / www . sciencedirect. com / science / article / pii/S1877042816000471.

[7] Bran Knowles and Vicki L. Hanson. The Wisdom of Older Technology (Non-)Users. Commun. ACM. (in press). 2018.

[8] Andreas Komninos, Emma Nicol, and Mark D. Dunlop. "Designed with Older Adults to SupportBetter Error Correction in SmartPhone Text Entry: The MaxieKeyboard". In: Proceedings of the 17th International Conference on Human-Computer Interaction with Mobile Devices and Services Adjunct. MobileHCI '15. Copenhagen, Denmark: ACM, 2015, pp. 797-802. ISBN: 978-1-4503-3653-6. DOI: 10 . 1145/2786567. 2793703, URL: http : / / doi . acm . org/ 10 . $1145 / 2786567.2793703$

[9] Chaiwoo Lee and Joseph F. Coughlin. "PERSPECTIVE: Older Adults' Adoption of Technology: An Integrated Approach to Identifying Determinants and Barriers". In: Journal of Product Innovation Management 32.5 (2015), pp. 747-759. ISSN: 1540-5885. DOI: 10 . 1111 / jpim . 12176 URL: http : / / dx . doi . org/10.1111/jpim.12176

[10] ExLibris Ltd. campusM. 2017. URL: http : / / wwW . campusm. com (visited on 10/19/2017).

[11] Jo Lumsden, Lilit Hakobyan, and Dympna O'Sullivan. "Participatory Design: How to Engage Older Adults in Participatory Design Activities". In: Int. J. Mob. Hum. Comput. Interact. 7.3 (July 2015), pp. 7892. ISSN: 1942-390X. DOI: 10 . 4018/ijmhci. 2015070106 URL: http://dx.doi.org/10. 4018/ijmhci.2015070106

[12] Mateusz Mikusz et al. "Repurposing Web Analytics to Support the IoT". In: Computer 48.9 (2015), pp. 42-49. ISSN: 0018-9162. DOI: $10.1109 / \mathrm{MC} .2015 .260$.

[13] Richard Mortier et al. "The personal container, or your life in bits”. In: Digital Futures 10 (2010), pp. 11-12.

[14] Ofcom. Connected Nations 2016 - 2G/3G Coverage. Accessed: 2017-10-18. 2016. URL: https : / / wWw . ofcom . org . uk / research and - data / multi - sector - research / infrastructure - research / connected nations - 2016 / interactive (visited on 12/16/2016).

[15] Ofcom. Rise of the Social Seniors revealed. Accessed: 2017-10-18. 2017. URL: https : / / www . ofcom . org . uk/about - ofcom/ latest / features and-news/rise-social-seniors (visited on 10/18/2017).
[16] Andraž Petrovčič et al. "Design of Mobile Phones for Older Adults: An Empirical Analysis of Design Guidelines and Checklists for Feature Phones and Smartphones". In: International Journal of Human-Computer Interaction (2017). (in press). DOI: 10 . 1080/ 10447318.2017 .1345142

[17] T. Phan, S. Kalasapur, and A. Kunjithapatham. "Sensor fusion of physical and social data using Web SocialSense on smartphone mobile browsers". In: 2014 IEEE 11th Consumer Communications and Networking Conference (CCNC). 2014, pp. 98-104. DOI: 10 . $1109 / \mathrm{CCNC} .2014 .6866555$

[18] Ivan Miguel Pires et al. "From Data Acquisition to Data Fusion: A Comprehensive Review and a Roadmap for the Identification of Activities of Daily Living Using Mobile Devices". In: Sensors 16.2 (2016). ISSN: 14248220. DOI: 10 . 3390/s16020184 URL: http: / / WWW.mdpi.com/1424-8220/16/2/184

[19] Lei Tian, Junqi Shi, and Zizhen Yang. "Why Does Half the World's Population Have a Mobile Phone? An Examination of Consumers' Attitudes toward Mobile Phones". In: CyberPsychology \& Behavior 12.5 (2009), pp. 513-516. DOI: $10.1089 / \mathrm{cpb} .2008 .0335$ URL: https://doi.org/10.1089/cpb.2008 . 0335 